\title{
Antibiomania after triple therapy for Helicobacter Pylori: Two case reports and a review of physiopathology
}

\author{
Aitor Miñambres*

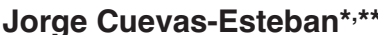 \\ Marta Pardo*** \\ Luisa Baladón $n^{\star, \star \star}$ \\ Marc Planella* \\ * Department of Psychiatry, Parc Sanitari \\ Sant Joan de Deu, Sant Boi de Llobregat, \\ Barcelona \\ ** Research and Development Unit, \\ CIBERSAM, Parc Sanitari Sant Joan de \\ Déu, Fundació Sant Joan de Déu, \\ St. Boi de Llobregat, Barcelona \\ *** Hospital Infanto-Juvenil Sant Joan de \\ Deu, Esplugues de Llobregat, Barcelona, \\ CIBERSAM
}

SPAIN

\begin{abstract}
Background and Objectives: The onset of manic symptoms in middle age requires clinicians to consider possible reversible causes, especially in patients with no previous psychiatric history. A number of drugs have been implicated as being among possible causes. The term antibiomania appeared to define cases of antibiotic-induced manic symptoms. This is a serious, but rare, adverse event. Several studies have described antimicrobial agents as being responsible for antibiomania. Our objective is to investigate the possible induction of manic symptoms by clarithromycin through two case reports and a review of the literature.

Methods: We report two cases of clinical manic psychotic symptoms arising in the context of treatment with triple therapy for Helicobacter pylori eradication. In addition, we summarize, in an unsystematic way, previously published evidence and pathophysiological mechanisms proposed.

Conclusions: These and other previously published cases suggest that the use of triple therapy, and especially of clarithromycin, should always be considered as a possible cause of acute manic or psychotic episode. Published evidence on the pathophysiological mechanisms is speculative so the identification and dissemination of a larger number of antibiomania cases and systematic study of them may help us to understand the underlying pathophysiological mechanisms and improve our diagnostic skills.
\end{abstract}




\section{Introduction}

The presence of antibiotic-associated psychiatric symptoms was described 60 years ago when several case reports of psychotic syndrome following penicillin administration emerged ${ }^{1}$. The term antibiomania subsequently appeared to define cases of antibiotic-induced manic symptoms. This is a serious, but rare, adverse event.

Regarding the number of antibiomania cases reported by the WHO and the FDA, clarithromycin seems to be the antibiotic most frequently implicated in the occurrence of this adverse effect, followed by ciprofloxacin and ofloxacin ${ }^{2}$.

The typical course of antibiomania is characterised by rapid onset of symptoms (usually within the first week of treatment) and quick, ad integrum remission after withdrawing antibiotics and administering antipsychotic medication, usually for a few days.

The aim of this paper is to report on two cases of manic episode after starting triple therapy to eradicate Helicobacter pylori (clarithromycin, amoxicillin and omeprazole) and to review the literature on pathophysiological mechanisms underlying this syndrome.

\section{Case report 1}

A 54-year-old man was admitted to our hospital because of manic symptoms and behavioral disorder.

There were no previous medical or psychiatric disorders or toxic abuse reported. Nor was there a family history of mental disorders. He had recently started treatment for Helicobacter Pylori (HP), taking $1 \mathrm{~g}$ of clarithromycin, 1 $\mathrm{g}$ of amoxicillin, and $20 \mathrm{mg}$ of omeprazole daily. The patient took the treatment for one week, and it was completed the day before hospitalisation. He had not been treated with any other drug in the previous 3 months.

The day before stopping triple therapy, he suddenly developed manic symptoms such as insomnia, hyperthymia, tachypsychia, verbiage and hyperactivity. He also showed messianic, jealous and paranoid delusions, as well as visual and auditory hallucinations (he described having seen and heard the Virgin Mary). He was conscious, oriented, and showing transitory hypertension $(210 / 120 \mathrm{mmHg})$ at admission, that immediately normalized after an isolated dosage of captopril $25 \mathrm{mg}$. Young mania rating scale score was 46 points and Mini-mental state examination score was 30 points out of 30 .There were no other remarkable results found during physical, imaging or laboratory examinations, including Blood Analysis (BA), Electrocardiogram (ECG) and Computerized Tomography (CT).

Risperidone $3 \mathrm{mg} / \mathrm{d}$ was started and the patient experienced rapid episode remission. The patient reported having taken omeprazole and amoxicillin in the past, but not clarithromycin. He was discharged 48 hours after admission with a diagnosis of Substance-Induced Mood Disorder (probably clarithromycin) and a score of 11 on the Young scale and 30/30 on the Mini-Mental State Exam (MMSE). Risperidone was maintained and gradually withdrawn over the following 10 days, verifying the absence of symptoms. Five years later, he was interviewed again. During this period, no manic or psychotic symptoms appeared. He did not take amoxicillin or clarithromycin during this period. 


\section{Case report 2}

A 47-year-old woman with no previous psychiatric or substance-abuse history was admitted to our psychiatric hospital because of behavioral disturbances and manic symptoms.

Her father was diagnosed as having a Schizoaffective disorder. Only well controlled hypertension and peptic ulcer disease were found relevant in her past medical history. She wasn't taking any regular medication.

One week before the onset of the episode, the patient was diagnosed as having an HP infection and she started taking $500 \mathrm{mg}$ of clarithromycin twice daily, $1 \mathrm{~g}$ of amoxicillin, and $40 \mathrm{mg}$ of omeprazole. Four days after starting this treatment, she suddenly developed insomnia, hyperactivity, grandiose delu- sions, irritability, pressured speech, tangential thinking and increased energy levels. These manic symptoms persisted and escalated and she was, consequently, hospitalised.

Her physical, laboratory and imaging examinations (including BA and CT) were unremarkable. Triple therapy was discontinued and olanzapine $20 \mathrm{mg}$ /day was administered from the day of admission. One week after admission, manic symptoms still persisted (insomnia, pressure speech, tachypsychia, hyperthymia, tangential thinking), although psychotic symptoms had improved a lot. Persistence of manic symptoms forced us to add Divalproex sodium $2000 \mathrm{mg} /$ day to her treatment. She was discharged after a three-week hospital stay. She was finally diagnosed with Bipolar I Disorder, Single Manic Episode, Severe With Psychotic Features (DSM-IV-TR).

Table 1

Comparison of clinical characteristics among case reports

CASE REPORT 1

Sex
Age
Personal Psychiatric History
Familial Psychiatric History
HP treatment

Hospital stay

Treatment

Treatment duration after discharge

Restitutio ad integrum

Diagnosis

Male

54

No

No

Amoxicillin $1 \mathrm{gr}$

Clarithromycin $1 \mathrm{gr}$

Omeprazole $20 \mathrm{mg}$

2 days

Risperidone $3 \mathrm{mg} /$ day

Progressive reduction

Stop in 9 days

Yes

Substance-induced mood disorder
CASE REPORT 2

Female

47

No

Yes

Amoxicillin $1 \mathrm{gr}$

Clarithromycin $1 \mathrm{gr}$

Omeprazole $40 \mathrm{mg}$

21 days

Olanzapine $20 \mathrm{mg} /$ day

Divalproex odium 2000 mg/day

Long-Term

No

Bipolar I disorder, single manic episode, severe with psychotic features

HP: Helicobacter Pylori. 


\section{Discussion}

In both cases, there is an absence of other concurrent medications or drug abuse and a lack of previous medical conditions or personal psychiatric history in the middle age of life. Also, physical, imaging, and laboratory examinations were completely normal. We did not carry out cerebral magnetic resonance imaging, spinal tap or electroencephalography because no clinical signs or symptoms indicated doing so. Considering that both patients were conscious and appropriately oriented, delirium was ruled out; Thus, triple therapy seemed the most probable cause attending to the chronological relationship.

Since our patients received a combination of three drugs (clarithromycin, amoxicillin, and omeprazole), we have to consider the possibility that any of the three (or combinations thereof) were potentially involved in the onset of symptoms. To the best of our knowledge, there are no manic reactions associated with omeprazole described in the literature or published elsewhere. Although there are isolated reports with amoxicillin, clarithromycin is the antibiotic most commonly associated with the occurrence of antibiomania $^{2}$. Therefore, we think of clarithromycin as the most probable inducer of both manic episodes. Nevertheless, only the reintroduction of each drug separately would fairly discriminate between them in light of the recurrence of symptoms. In this sense, and in relation to case report \#1, the previous use of amoxicillin and omeprazole without development of any mental disturbance strengthens our hypothesis.

There are no known interactions between amoxicillin and omeprazole, or amoxicillin and clarithromycin. Mutual interactions between omeprazole and clarithromycin have been described, showing raised levels of both drugs in blood plasma ${ }^{3}$. This situation could lead to an increase in clarithromycin crossing the blood-brain barrier, allowing for the presence of neuropsychiatric symptoms.

Despite similarities between the two patients, some features are clearly different in the two cases and point to different diagnoses.

In the first case, negative family history for mental disorders, fast remission after withdrawal of antibiotic using low risperidone dosage, and absence of relapse after years without psychopharmacological treatment suggest a substance-induced mood disorder.

On the other hand, distinct features can be seen in the second case. There is a family history of schizoaffective disorder and slower and poorer improvement using antipsychotics after withdrawing the antibiotic, so the clinician finally required the use of a mood stabilizer. Moreover the patient did not show complete remission of symptoms after discharge and needed long-term psychopharmacological treatment. In the reported clarithromycin-induced manic episodes, a complete resolution of symptoms 24 to 36 hours after completing treatment is described ${ }^{2}$. All these features suggest the onset of bipolar disorder, possibly induced by triple therapy treatment.

In general terms, the first therapeutic approach in the presence of suspicion of antibiomania should be the withdrawal of the drug, adding, if indicated, low doses of antipsychotics to achieve clinical remission. Although antipsychotic drugs were immediately started in both reported cases, one could argue whether symptoms would have improved just by discontinuing triple therapy. It seems possible in the first case, but not in the second one.

Persistence of symptoms despite these measures may indicate the onset of bipolar disorder. Even if complete remission is achieved, the risk of development of a true bipolar disorder should always be considered throughout the patient's lifetime. 
There is little published scientific evidence on pathophysiological mechanisms and what there is speculative. This situation brings us back to the primary affective disorder pathophysiology hypothesis.

\section{Antibiomania physiopathology}

Various proposals were found in the literature to explain the pathophysiology of this phenomenon.

- GABA: Antagonism of the GABA system has been proposed as the mechanism whereby antibiotics induce mania. The GABAergic deficit causes dopaminergic hyperactivity, especially on D2 receptors, related to the appearance of positive symptoms in schizophrenia. Quinolones competitively inhibit the binding of GABA to its receptors ${ }^{4}$ and Isoniazid decreases GABA-mediated transmission, increasing levels of neurosteroids in the $\mathrm{CNS}^{5}$. Nevertheless, we did not find any evidence in the literature of a possible effect of clarithromycin or amoxicillin on the GABA system.

- CORTISOL: Alterations in cortisol level underlying depression and mania are well known. An elevation of cortisol levels in patients with acute manic episode has been described $^{6}$.

Rifampicin has been related to altered Dexamethasone suppression tests in patient with affective disorders ${ }^{7}$. It has been reported that rifampicin induces adrenal crises in patients with Addison's disease on corticosteroid replacement therapy ${ }^{8}$. Rifampicin may accelerate corticosteroid metabolism and clearance, thanks to CYP3A4 induction and to increased urinary excretion of 6-hydroxy$\operatorname{cortisol}^{9,10}$. Moreover, Rifampicin was proposed as a possible trigger of glucocorticoid receptors $(\mathrm{GR})^{11}$, which could explain the adverse effects of the drug on the CNS. Nevertheless, another study failed to demonstrate GR activation by rifampicin in some tissues afterwards $^{12}$.

Macrolides, such as clarithromycin, slow glucocorticoid clearance ${ }^{13}$. Concomitant use of clarithromycin results in a significantly higher plasma concentration and a $65 \%$ reduction in methylprednisolone elimination ${ }^{14}$.

- GLUTAMATE: It has been postulated that macrolides may cause psychosis-like symptoms due to their inhibitory action on the glutamate system through NMDA receptors in the CNS ${ }^{15}$.

- PROSTAGLANDINS: Increased levels of PGE1 in patients with manic episodes have been described. Lithium normalises PGE1 levels inhibiting its precursor, dihomogamma-linolenic acid ${ }^{16,17}$. PGE-1 is related to calcium uptake regulation and intracellular release. They are crucial to nerve conduction mechanisms and regulation of transmitter release ${ }^{18}$. There is increasing interest in the relationship between the arachidonic acid system (AA) and the pathophysiology of bipolar disorder. It has been described as an AA pathological excess, along with an increase in PGE2, its final product, in bipolar disorder ${ }^{19}$. The therapeutic action of antimanic-mood stabilisers (lithium, carbamazepine and valproate) is related to decreased AA and PGE COX-2 cerebral release ${ }^{20}$. On the other hand, other authors have not observed such a relationship between levels of PGEs and mania ${ }^{21,22}$.

Several studies suggest that a wide range of antibiotics (some quinolones, cephalosporins, tetracyclines, rifampicin, clindamycin and some macrolids including clarithromycin) could modify levels of PGE-2 in several tissues $^{23-33}$. 
- OTHER PROPOSITIONS: There is growing evidence on several intracellular pathways involved in bipolar disorder pathophysiology, including the Akt and Wnt system, through GSK-3B protein. The therapeutic effect of lithium and valproate has been linked to its GSK-3B inhibition ${ }^{34-36}$. The macrolide rapamycin could act through inhibition of epileptogenesis, as well as its action on Akt and GSK-3b mTORC and on mitochondrial protein synthesis ${ }^{37}$. To the best of our knowledge, there is no similar evidence regarding clarithromycin or amoxicillin.

There is also growing information suggesting that individual genetic factors could play a role in the risk for developing neural side effects of $\operatorname{a~drug}^{38}$. There is no published evidence regarding antibiomania, but this possibility might help in the understanding of this phenomenon in the future.

It has recently been suggested that gut microbiota could play a role in maintaining homeostasis in health and contributing to the pathogenesis of some diseases, including depression. In fact, some information points to a possible different pattern of gut microbiota in depression and stress-models in animals ${ }^{39}$. The term "psychobiotics" has recently been coined to denote live organisms (e.g., probiotics) that, when ingested in adequate amounts, could improve some mental health diseases. Preclinical work suggests that certain psychobiotics possess antidepressant activity in animal models. Effects are thought to be mediated via the vagus nerve, spinal cord, or neuroendocrine systems ${ }^{40}$. Although this is highly hypothetical, as short-treatment with clarithromycin produces modifications of the gut microbiota immediately after treatment ${ }^{41}$, one may speculate that these changes may be a possible explanation for the mood disturbances in antibiomania.

\section{Conclusions}

Most antibiomania cases are relatively recent. This could be because this adverse effect is more common among newer antimicrobials, but could also be explained by the current emphasis on adverse effects and greater knowledge of them.

These and other previously published cases suggest that the use of triple therapy, and especially of clarithromycin, should always be considered as a possible cause of acute manic or psychotic episode. Published evidence on the pathophysiological mechanisms is speculative so the identification and dissemination of a larger number of antibiomania cases and systematic study of them may help us to understand the underlying pathophysiological mechanisms and improve our diagnostic skills.

\section{References}

1. Cohen SB. Psychosis resulting from penicillin hypersensitivity; report of a case and review of the literature. Am J Psychiatry 1955; 111(9): 699-702.

2. Abouesh A, Stone C, Hobbs WR. Antimicrobial-induced mania (antibiomania): a review of spontaneous reports. J Clin Psychopharmacol 2002; 22(1): 71-81.

3. Gustavson LE, Kaiser JF, Edmonds AL, Locke CS, DeBartolo ML, Schneck DW. Effect of omeprazole on concentrations of clarithromycin in plasma and gastric tissue at steady state. Antimicrob Agents Chemother 1995; 39(9): 2078-2083.

4. Tsuji A, Sato H, Kume Y, Tamai I, Okezaki E, Nagata $\mathrm{O}$, et al. Inhibitory effects of quinolone antibacterial agents on gamma-aminobutyric acid binding to receptor sites in rat brain membranes. Antimicrob Agents Chemother 1988; 32(2): 190-194.

5. Barbaccia ML, Roscetti G, Trabucchi M, Purdy RH, Mostallino MC, Perra C, et al. Isoniazid-induced inhibition of GABAergic transmission enhances neurosteroid content in the rat brain. Neuropharmacology 1996; 35(9-10): 12991305. 
6. Daban C, Vieta E, Mackin P, Young AH. Hypothalamic-pituitary-adrenal axis and bipolar disorder. Psychiatr Clin North Am 2005 Jun; 28(2): 469-80.

7. Kyriazopoulou V, Vagenakis AG. Abnormal overnight dexamethasone suppression test in subjects receiving rifampicin therapy. J Clin Endocrinol Metab 1992; 75: 315-317.

8. Kyriazopoulou V, Parparousi O, Vagenakis AG. Rifampicin-induced adrenal crisis in addisonian patients receiving corticosteroid replacement therapy. J Clin Endocrinol Metab 1984; 59: 1204-1206.

9. Park BK, Breckenridge AM. Clinical implications of enzyme induction and enzyme inhibition. Clin Pharmacokinet 1981; 6: 1-24.

10. Yamada S, Iwai K. Induction of hepatic cortisol-6-hydroxylase by rifampicin. Lancet 1976; 2: 366-367.

11. Calleja C, Pascussi JM, Mani JC, Maurel P, Vilarem MJ. The antibiotic rifampicin is a nonsteroidal ligand and activator of the human glucocorticoid receptor. Nat Med 1998; 4: 92-95.

12. Herr AS, Wochnik GM, Rosenhagen MC, Holsboer F, Rein T. Rifampicin is not an activator of glucocorticoid receptor. Mol Pharmacol 2000; 57(4): 736-737.

13. Ushiama H, Echizen H, Nachi S, Ohnishi A. Dose-dependent inhibition of CYP3A activity by clarithromycin during Helicobacter pylori eradication therapy assessed by changes in plasma lansoprazole levels and partial cortisol clearance to 6beta-hydroxycortisol. Clin Pharmacol Ther 2002; 72(1): 33-43.

14. Fost DA, Leung DYM, Martin RJ, Brown EE, Szefler SJ, Spahn JD. Inhibition of methylprednisolone elimination in the presence of clarithromycin therapy. J Allergy Clin Immunol 1999; 103: 1031-1035.

15. Manev H, Favaron M. Macrolide antibiotics protect neurons in culture against the $\mathrm{N}$-methyl-D-aspartate receptor-mediated toxicity of glutamate. Brain Res 1993; 624: 331-335.

16. Horrobin DF. Lithium as a regulator of prostaglandin synthesis: relevance to manic depressive psychosis and schizophrenia and to the mechanism of opiate action. In: Cooper T, Kline NS, Schou M, eds. Lithium: an international conference. Amsterdam: Excerpta Medica (in press).

17. Manku MS, Horrobin DF, Karmazyn M, Cunnane SC. Prolactin and zinc effects on rat vascular reactivity: possible relationship to dihomogammalinolenic acid and to prostaglandin synthesis. Endocrinology 1979; 104: 774-779.

18. Horrobin DF, Manku MS. Possible role of prostaglandin E1 in the affective disorders and in alcoholism. Br Med J 1980; 280(6228): 1363-1366.
19. Rapoport SI, Basselin M, Kim HW, Rao JS. Bipolar disorder and mechanisms of action of mood stabilizers. Brain Res Rev 2009; 61: 185-209.

20. Rao JS, Ertley RN, Lee HJ, Rapoport SI, Bazinet RP. Chronic fluoxetine upregulates activity, protein and mRNA levels of cytosolic phospholipase A2 in rat frontal cortex. Pharmacogenomics J 2006; 6(6): 413-420.

21. Sublette ME, Bosetti F, DeMar JC, Ma K, Bell JM, Fagin-Jones S, et al. Plasma free polyunsaturated fatty acid levels are associated with symptom severity in acute mania. Bipolar Disord 2007; 9(7): 759-765.

22. Gerner RH, Merrill JE. Cerebrospinal fluid prostaglandin $\mathrm{E}$ in depression, mania, and schizophrenia compared to normals. Biol Psychiatry 1983; 18(5): 565-569.

23. Corps AN, Curry VA, Harrall RL, Dutt D, Hazleman BL, Riley GP. Ciprofloxacin reduces the stimulation of prostaglandin $\mathrm{E}(2)$ output by interleukin-1beta in human tendon-derived cells. Rheumatology 2003; 42(11): 1306-1310.

24. Fukuishi N, Matsui N, Kanoh R, Akagi M. Novel action of quinolones on osteoclast-like cells. J Toxicol Sci 1999; 24(5): 383-391.

25. Takahashi HK, Iwagaki H, Xue D, Katsuno G, Sugita $\mathrm{S}$, Mizuno K, et al. Effect of ciprofloxacin-induced prostaglandin E2 on interleukin-18-treated monocytes. Antimicrob Agents Chemother 2005; 49(8): 3228-3233.

26. Akanuma S, Hosoya K, Ito S, Tachikawa M, Terasaki $\mathrm{T}$, Ohtsuki S. Involvement of multidrug resistance-associated protein 4 in efflux transport of prostaglandin $E(2)$ across mouse blood-brain barrier and its inhibition by intravenous administration of cephalosporins. J Pharmacol Exp Ther 2010; 333(3): 912-919.

27. Silva Bastos LF, Pinheiro de Oliveira AC, Magnus Schlachetzki JC, Fiebich BL. Minocycline reduces prostaglandin E synthase expression and 8-isoprostane formation in LPS-activated primary rat microglia. Immunopharmacol Immunotoxicol 2011; 33(3): 576-580.

28. Kim SS, Kong PJ, Kim BS, Sheen DH, Nam SY, Chun W. Inhibitory action of minocycline on lipopolysaccharide-induced release of nitric oxide and prostaglandin E2 in BV2 microglial cells. Arch Pharm Res 2004; 27(3): 314-318.

29. Patel RN, Attur MG, Dave MN, Patel IV, Stuchin SA, Abramson SB, et al. A novel mechanism of action of chemically modified tetracyclines: inhibition of COX-2-mediated prostaglandin E2 production. J Immunol 1999; 163(6): 3459-3467.

30. Spreer A, Lugert R, Stoltefaut V, Hoecht A, Eiffert H, Nau R. Short-term rifampicin pretreatment reduces inflammation and neuronal cell death in a rabbit model of bacterial meningitis. Crit Care Med 2009; 37(7): 2253-2258. 
31. Yuhas Y, Azoulay-Alfaguter I, Berent E, Ashkenazi S. Rifampin inhibits prostaglandin E2 production and arachidonic acid release in human alveolar epithelial cells. Antimicrob Agents Chemother 2007; 51(12): 4225-4230.

32. Sato T, Shirane T, Noguchi N, Sasatsu M, Ito A. Novel anti-acne actions of nadifloxacin and clindamycin that inhibit the production of sebum, prostaglandin E(2) and promatrix metalloproteinase-2 in hamster sebocytes. J Dermatol 2012; 39(9): 774-780.

33. Miyazaki M, Zaitsu M, Honjo K, Ishii E, Hamasaki Y. Macrolide antibiotics inhibit prostaglandin E2 synthesis and mRNA expression of prostaglandin synthetic enzymes in human leukocytes. Prostaglandins Leukot Essent Fatty Acids 2003; 69(4): 229-235.

34. Klein PS, Melton DA. A molecular mechanism for the effect of lithium on development. Proc Natl Acad Sci USA 1996; 93: 8455-8459.

35. Kaladchibachi SA, Doble B, Anthopoulos N, Woodget JR, Manoukian AS. Glycogen synthase kinase 3, circadian rhythms, and bipolar disorder: a molecular link in the therapeutic action of lithium. J Circadian Rhythms 2007; 5: 3.

36. Meijer L, Flajolet M, Greengard P. Pharmacological inhibitors of glycogen synthase kinase 3. Trends Pharmacol Sci 2004; 25: 471-480.

37. Khalil RB. Is there any place for macrolides in mood disorders?. Med Hypotheses 2012; 78(1): 86-87.
38. Lu DY, Lu TR, Zhu PP. Genetics in neural toxicities of drugs. Cent Nerv Syst Agents Med Chem 2012; 12(4): 250-253.

39. Dinan TG, Cryan JF. Melancholic microbes: a link between gut microbiota and depression? Neurogastroenterol Motil 2013; 25(9): 713-719.

40. Dinan TG, Stanton C, Cryan JF. Psychobiotics: a novel class of psychotropic. Biol Psychiatry 2013; 74(10): 720-726.

41. Jakobsson HE, Jernberg C, Andersson AF, SjölundKarlsson M, Jansson JK, Engstrand L. Short-term antibiotic treatment has differing long-term impacts on the human throat and gut microbiome. PLoS One 2010; 5(3): e9836.

\author{
Corresponding author: \\ Jorge Cuevas-Esteban \\ Parc Sanitari Sant Joan de Deu \\ Unidad de Agudos de Psiquiatría \\ Doctor Antoni Pujadas, 42. \\ 08830. Sant Boi de Llobregat \\ Barcelona - Spain \\ Teléfono: 936406350 \\ Fax: 936542136 \\ E-mail: jm.cuevas@pssjd.org
}

